\title{
Image Enhancement of Underexposed and Overexposed Images using Tanlog Technique
}

\author{
$\operatorname{Varsha}^{1,2}$, Manju Mathur ${ }^{1,2}$ \\ ${ }^{1}$ Rajasthan College Of Engineering For Women, Bhankrota, Jaipur \\ ${ }^{2}$ Department of Electronics and Communication Engineering, RCEW, Jaipur
}

\begin{abstract}
Now a day's applications require various kinds of images as sources of information for interpretation and inspection. Image enhancement is technique of put on altered modifications to an input image to create the subsequent image more attractive or to deliver an improved renovate production for upcoming automated image processing techniques. Image enhancement of any underexposed or overexposed image are critically crucial to fetch the information as extracting information is very difficult and sometimes this information is completely lost. There have been few papers which discusses the image enhancement algorithms using the fusion technique of underexposed and overexposed images. However the demand of overexposed and underexposed images might be difficult or sometimes it may be impossible to gather such images for exact same frame. In this paper an algorithm has been proposed which uses either overexposed image or underexposed image and still improves the quality of the image. Algorithm will work on spatial domain operation and frequency domain operation as well.
\end{abstract}

Keywords: Tanlog Equation, Median filter, Gamma correction, Histogram Equalisation, Homomorphic Filter, CDF

\section{Introduction}

There is no general concept of image enhancement. When an image is managed for pictorial elucidation, the spectator is the eventual critic of how well a specific process works. Pictorial evaluation of image superiority is a highly idiosyncratic process, thus making the demarcation of a "good image" an vague standard by which to associate algorithm performance. When the difficulty is one of handling images for device perception, the valuation job is slightly easier. For specimen, in dealing with a character appreciation presentation, and parting aside other matters such as computational requirements, the best image processing method would be the one yielding the best machine recognition results

Image enhancement is the process of transforming digital images so that the results are more suitable for display or further image analysis. These methods have been widely used in many applications of image processing where the subjective quality of images is important for human construal. In any individual assessment of image quality Contrast is an important feature. Contrast is created by the difference in luminance reflected from two adjacent surfaces. In other words, contrast is the difference in visual properties that makes an object distinguishable from other objects and the background. In visual perception, contrast is determined by the difference in the color and brightness of the object with other objects. Our visual system is more sensitive to contrast than absolute luminance; therefore, we can perceive the world similarly regardless of the considerable changes in illumination conditions. Many algorithms for accomplishing contrast enhancement have been developed and applied to difficulties in image processing.

Image enhancement is one of the pre-proceeding performances in digital image processing. Image enhancement operation can improve the digital image quality in terms of contrast, noise reduction, brightness, etc. Besides as improving the quality of the image to be displayed, the image enhancement results might be used for further image analysis process, in this case as preprocessing. Image enhancement can overcome the objects of the image that is difficult to distinguish in the image due to the influence of luminance or noise.

Many contrast enhancement algorithms have been developed for many years with different approaches as needed. Morphological contrast enhancement algorithm to boost the image contrast using morphological method is planned. Image contrast enhanced by adding a bright area and subtracting the dark areas so that the features of the object in the image become sharper. Sharp features of the objects could be enhancing the analysis results in terms of image segmentation. The proposed method is associated to the contrast enhancement with HE(histogram equalization).In image contrast enhancement Histogram equalization is one of the most standard methods [1]. The cluster validity of image segmentation was tested using silhouette coefficient method. The contrast enhancement algorithm is measured based on the quality of the clusters shown after the segmentation process.

\section{Literature Survey}

\section{Histogram Equalization}

Histogram equalization (HE) as in [2] is the well-known or usually used technique for contrast enhancement because of its simplicity and comparatively better performance on almost all types of images. Histogram Equalization constructed on the gray levels remapping of the image based on the $\mathrm{PD}$ (probability distribution) of the input gray levels. HE methods can be classified in two principle categories- global and local histogram equalization. 


\section{International Journal of Science and Research (IJSR) \\ ISSN (Online): 2319-7064}

Index Copernicus Value (2016): 79.57 | Impact Factor (2015): 6.391

Global Histogram Equalization (GHE) customs the histogram information of the complete input image for its renovation purpose. However, this inclusive methodology is appropriate for complete enhancement; it flops to adjust with the local brightness features of the input image. Gray levels with very high frequencies, rule the other gray levels having lower frequencies and in such a situation, remapping of gray level is done by Global histogram Equalization(GHE) in a way that the contrast widening converts restricted in some dictating gray levels having greater image histogram constituents and causes prevailing contrast loss for other small ones.

LHE (Local histogram equalization) can be unrestricted from such problem. It practices a minor window that glides through every pel of the image serially and individual the block of pels that drop in this window are taken into interpretation for HE and then gray level mapping for enhancement is done only for the center pixel of that window. Hence local information get appropriately used.LHE has some disadvantages: (i) it needs high computational cost; (ii) it sometimes causes overenhancement in some portion of the image; (iii) it enhances the noises in the input image along with the image features. To reduce high computational cost, alternative methodology is to smear non-overlapping block grounded HE. Although these procedures yield and is agreeable checkerboard effects on enhanced images [2,3].

In [4] authors proposed an extended form of bi-histogram equalization called bi-histogram equalization with neighborhood metric (BHENM). BHENM consisted of two stages. First, large histogram bins that caused washout artifacts were divided into sub-bins using neighborhood metrics; the same intensities of the original image were arranged by neighboring information. In next stage, based on the mean of the histogram of the original image the original image had been divided into two sub histograms; now they were equalized independently using refined histogram equalization, which produced flatter histograms. In an experimental trial, BHENM simultaneously cared for the brightness and enhanced the local contrast of the original image. on the other hand the limitation is that the execution time of this method was three times higher than that of GHE.

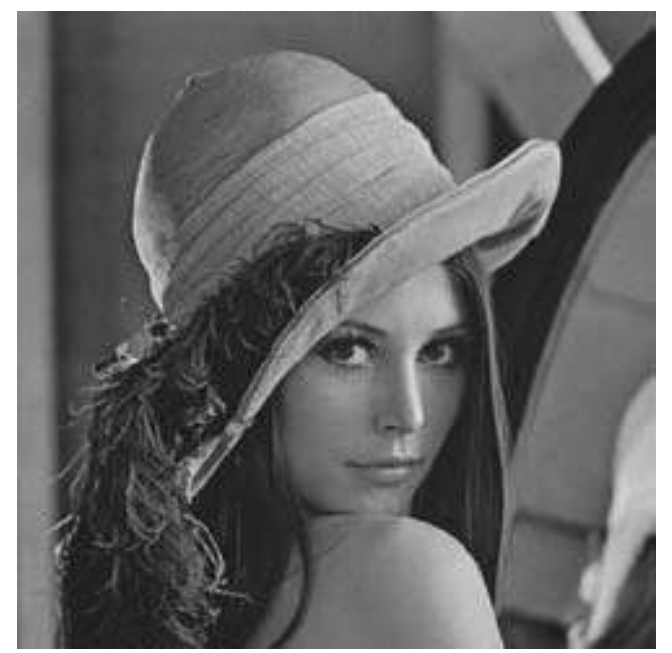

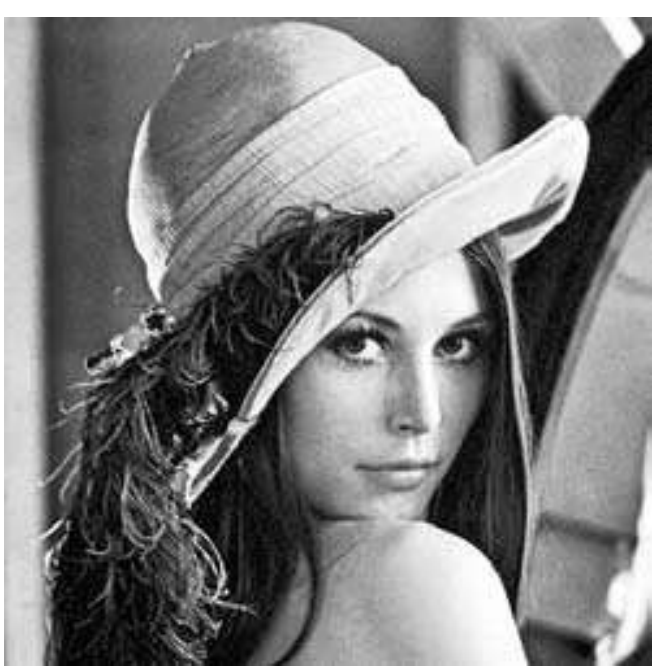
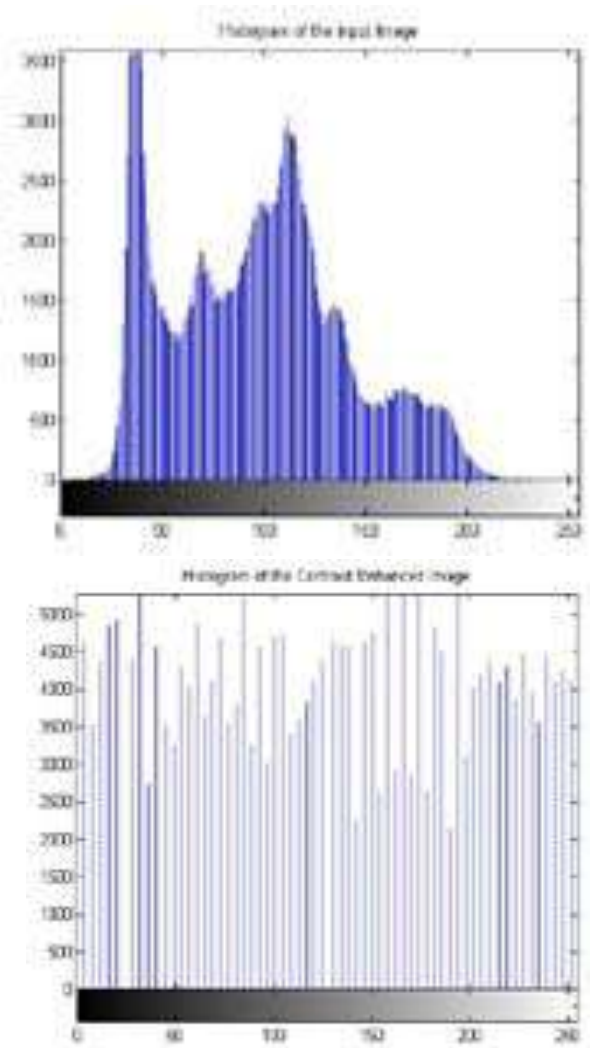

Figure 1: (a) Input Image (b) Histogram Equalized Image (c) Histogram of Input Image (d) Histogram of Equalized Image

\section{Fuzzy Technique}

In Fuzzy image every pel of a image is handled on the account of guidelines practical to the pels. 


\section{International Journal of Science and Research (IJSR) \\ ISSN (Online): 2319-7064}

Index Copernicus Value (2016): 79.57 | Impact Factor (2015): 6.391

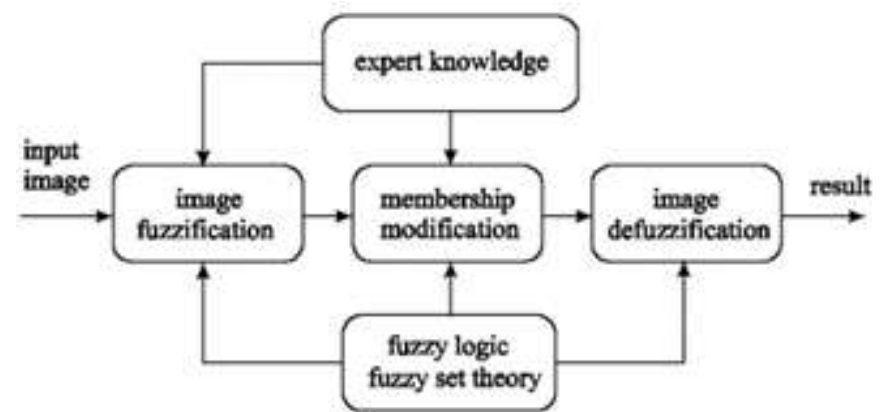

Figure 2: Phases in Fuzzy Enhancement

A Gray Image pixel comprises 256 intensity levels. So membership functions which are used for improving Intensity component of a pixel can be distinct in the universe of discourse $[0, . ., 255][5]$. Let $\mathrm{S}\left(n_{1}, n_{2}\right)$ be the variable on behalf of the intensity value of the pixel of coordinates $\left(n_{1}, n_{2}\right)$ in the source image and $\operatorname{fv}\left(n_{1}, n_{2}\right)$ be the conforming fuzzy variable in the resulting image after being enhanced.

IF $\mathrm{S}\left(n_{1}, n_{2}\right)$ is DARK, then $\operatorname{fv}\left(n_{1}, n_{2}\right)$ is BRIGHT.

IF $\mathrm{S}\left(n_{1}, n_{2}\right)$ is MED, then $\operatorname{fv}\left(n_{1}, n_{2}\right)$ is MED.

IF $\mathrm{S}\left(n_{1}, n_{2}\right)$ is BRIGHT, then $\operatorname{fv}\left(n_{1}, n_{2}\right)$ is DARK.

\section{Gamma Correction}

A non-linear transfer of signal lies between an electrical device and an optical device. This non linearity causes serious attenuation in the intensity of an image, ensuing with definite parts being too dark and with certain parts being faded out. To compensate for this non-linearity, an image processing algorithm called gamma correction is applied to the video signal to produce an image with fidelity. So Gamma is an significant representative of all digital imaging systems which describes the relation between a pixel value and its actual luminance. Gamma correction is a non-linear transformation used to regulate the illumination and contrast of an image. It is used in image processing to recompense for non-linear relations in imaging sources, display and printers. It not only varies the overall brightness of an image but also the proportion of red to blue to green. If images are not properly corrected, they are of poor quality and have reduced predictability of texture and colors.

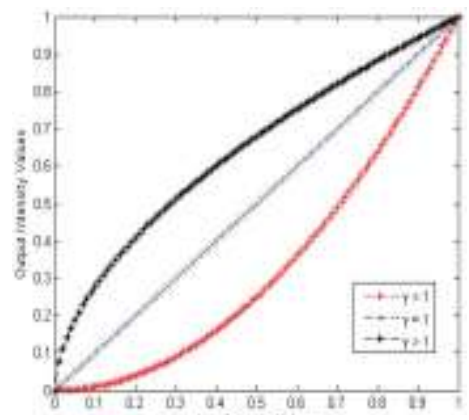

Fig. 3: Gamma Curves

The gamma correction [4] function is a monotonic function producing output pixel intensity, $\mathrm{X}_{(\mathrm{i}, \mathrm{j})}$ which is proportional to inverse of gamma power of input pixel intensity, $\mathrm{x}_{(\mathrm{i}, \mathrm{j})}$ and is defined as :-

$$
X(i, j)=255 *\left(\frac{x(i, j)}{255}\right)^{\frac{1}{\gamma}}
$$

Figure (3) shows the gamma curves. It can be observed that the image corrected image is overexposed than the original image for $\gamma<1$ and is underexposed than original image for $\gamma$ $>1$.

\section{Median Filter}

Median filtering is a nonlinear scheme which is used to eradicate noise from images. It is widely used as it is very effective at removing noise while preserving edges. It is particularly effective at removing 'salt and pepper' type noise. The median filter works by moving through the image pixel by pixel, replacing each value with the median value of neighboring pixels. The pattern of neighbors is called the "window", which slides, pixel by pixel over the entire image 2 pixel, over the entire image. The median is premeditated by principally organizing all the pixel values from the window into arithmetical order, and then substituting the pixel being considered with the median (middle) pixel value.

\section{Homomorphic Filtering}

The typical filter for homomorphic filtering process has been introduced in [1]- [5]. This filter has circularly symmetric curve shape, centered at $(u, v)=(0,0)$ coordinates in frequency domain. This filter is adapted from Gaussian high-pass filter, which is known as DoG (Difference of Gaussian) filter. The transfer function for DoG filter is defined as:

$$
H(u, v)=\left(\gamma_{H}-\gamma_{L}\right)\left[1-\exp \left\{-c\left(\frac{D(u, v)}{D_{0}}\right)^{2}\right\}\right]+\gamma_{L}
$$

where constant $c$ has been familiarized to control the steepness of the slope, $D 0$ is the cut-off frequency, $D(u, v)$ is the distance between coordinates $(u, v)$ and the Centre of frequency at $(0,0)$. For this filter, three main constraints are required to be set by the user. They are the high frequency gain $\gamma_{H}$, the low frequency gain $\gamma_{L}$,and the cut-off frequency $D_{0}$. If $\gamma_{H}$ is set greater than 1 , and $\gamma_{L}$ is set lower than 1 , the filter function inclines to diminution the involvement finished by the brightness (which occupies mostly the low frequency components) and amplify the influence prepared by the reflectance (which occupies most of the high frequency components). At the end, the net result will be a instantaneous dynamic range compression and contrast enhancement. The value of the low frequency gain should be set such as $\gamma_{L}=0.5$, to halve the spectral energyof the illumination, and the value of high frequency gain is set such as $\gamma_{H}=2$ to double the spectral energy ofthe reflectance components [1]. In [6], the value of $c$ is suggested to be equal to 0.5 . In practice, all these three parameter values are often determined empirically and there is no clear way to choose the exact suitable values for these parameters.

\section{Volume 6 Issue 12, December 2017}

\section{www.ijsr.net}




\section{International Journal of Science and Research (IJSR) \\ ISSN (Online): 2319-7064 \\ Index Copernicus Value (2016): 79.57 | Impact Factor (2015): 6.391}

\section{Methodology/ Proposed Work}

Image Enhancement can be done in two modes :- the first one can be generalized as Visual appearance based image enhancement and the second mode can be generalized as Entropy extraction based image enhancement. In this research work both the modes are used parallel. The proposed algorithm can be divided into two phases:- phase one consist of spatial domain operations and the phase two consist of frequency domain operations. Along with this a manual histogram equalization process has also been incorporated. In the proposed algorithm a RGB image will be taken as an input and will be analyzed over the different parameters such as Entropy of an image, composite average of an image and the component average of the image. After calculating the parameters image will be exposed to point operation. Point operation will take an image of the format of RGB and will separate out the RGB components and an equation will be implied over the three components. For point operation an equation has been used which can be termed as cos log equation, it will serve as a first point equation for the proposed algorithm

$$
u(x, y)=(\operatorname{cosd}(\log (f(x, y)) * \cos d(255)) * 255
$$

Along with the cosd $\log$ equation, tand $\log$ equation is also used. Tand log equation is as follows:-

$$
\begin{gathered}
u(x, y)=\operatorname{tand}(\log (R(x, y))) * \operatorname{tand}(255) \\
f u x(x, y)=u(x, y) * 255
\end{gathered}
$$

Where $\mathrm{u}(\mathrm{x}, \mathrm{y})$ is operated image matrix, $\mathrm{R}(\mathrm{x}, \mathrm{y})$ is the red component of the input image, fux $(\mathrm{x}, \mathrm{y})$ is the final operated image.

After the point operation the image will be passed through image contrast enhancement equation, which is as follows :

$$
\mathbf{a}(\mathbf{x}, \mathbf{y})=\frac{\mathbf{a}_{\mathbf{0}(\mathrm{x}, \mathrm{y})}-\mathbf{M}_{\mathbf{1}}}{\mathbf{M}_{\mathbf{2}}-\mathbf{M}_{\mathbf{1}}} * \mathbf{M}_{\mathrm{g}}
$$

Where $\mathrm{a}(\mathrm{x}, \mathrm{y})$ is operated image matrix, $a_{0}(\mathrm{x}, \mathrm{y})$ is the component of the image input, $M_{1}$ is the minimum value of the single component of input image, $M_{2}$ is the maximum value of the single component of input image, $M_{g}$ is the maximum gray levels on the side lobes of the current picture. By changing the contrast value of an image, changes the range of luminance values present in the image.

After the image contrast enhancement apply mask operation which will consist of median filtering, gamma correction and manual histogram equalization.

Median filter are statistical non-linear filters that are often described in the spatial domain. A Median filter smoothens the image by utilizing the median of the neighbourhood. Median filter performs the following tasks to find out each pixel value in the processed image:

1) All pixels in the neighbourhood of the pixel in the original image which are identified by the mask are sorted in the ascending or descending order.

2) The median of the sorted value is computed and is chosen as the pixel value for the processed image.

Gamma correction is the name of a nonlinear operation used to encode and decode luminance in video or image systems. Gamma correction can be defined by the following power law expression:

$$
X(i, j)=255 \times\left(\frac{x(i, j)}{255}\right)^{\frac{1}{r}}
$$

It can be observed that the corrected image is overexposed than the original image for $\gamma<1$ and is underexposed than original image for $\gamma>1$.

Manual Histrogram Equalization basically improves the histogram (plot of number of occurrence of gray levels in the image against the gray level values ) of an input image so as to improve the visual quality of the image. This technique reassigns the brightness values of pixels based on the image histogram. It is a technique where the histogram of the resultant image is as flat as possible. Manual Histrogram Equalization is done by performing the following steps:

1) Find the running sum of the Histogram values.

2) Normalize the values from step (1) by dividing the total number of pixels.

3) Multiply the values from step (2) by the maximum graylevel value and round.

4) Map the gray level values to the results from step (3) using a one to one correspondence.

Process will now enter into the frequency domain of the input image. Homomorphic filtering will be applied. Homomorphic filtering is used to remove multiplicative noise. An image can be modeled as the product of an illumination function and reflectance function at every point. Homomorphic filtering contains these following steps:

1) First of all logarithm of the image intensity taken so that these multiplicative components of the image can be separated linearly in the frequency domain.

2) After step (1) Fourier Transform is taken.

3) Then the desired homomorphic filter function $\mathrm{H}(\mathrm{k}, \mathrm{l})$ is applied separately to the illumination and reflectance components respectively.

4) In order to visualize the image, Inverse Fourier Transform followed by exponential function is applied.

Working of homomorphic filter on an input image is shown below:

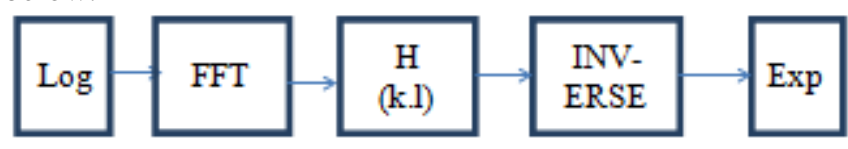

Volume 6 Issue 12, December 2017

www.jijsr.net

Licensed Under Creative Commons Attribution CC BY 


\section{International Journal of Science and Research (IJSR) \\ ISSN (Online): 2319-7064 \\ Index Copernicus Value (2016): 79.57 | Impact Factor (2015): 6.391}

Homomorphic filter will be applied only on the Underexposed image to filter out the low frequency band.

Now CDF (Cumulative Distribution Function) will be applied on both type of images (overexposed and underexposed). The cumulative distribution function of a variable $X$ is the function given by

$$
\mathrm{F}_{\mathrm{X}}(\mathrm{x})=\mathrm{P}(\mathrm{X}<=\mathrm{x}),
$$

Where $\mathrm{P}(\mathrm{X}<=\mathrm{x})$ represents the probability that the random variable $X$ takes on a value less than or equal to $x$. The probability that $\mathrm{X}$ lies in the semi closed interval $(a, b)$ where $a<\mathrm{b}$, is therefore

$$
\mathrm{P}(\mathrm{a}<\mathrm{X}<=\mathrm{b})=\mathrm{F}_{\mathrm{X}}(\mathrm{b})-\mathrm{F}_{\mathrm{X}}(\mathrm{a}) .
$$

The CDF of a continuous random variable $X$ can be expressed as the integral of its probability density function $f_{\mathrm{X}}$ as follows:

$$
F_{X}(x)={ }_{-\infty}^{x} \int f_{x}(t) d t .
$$

In the case of a random variable $X$ which has distribution having a discrete component at a value $b$,

$$
\mathrm{P}(\mathrm{X}=\mathrm{b})=\mathrm{F}_{\mathrm{X}}(\mathrm{b})-\lim _{-} \mathrm{x} \rightarrow \mathrm{b} \mathrm{F}_{\mathrm{X}}(\mathrm{x}) .
$$

If $F_{X}$ is continuous at $b$, this equals zero and there is no discrete component at $b$.

\section{Results}

The image enhancement algorithm presented here represents the image recovered after the application of certain equations which are explained above in the proposed methodology section. The tables here shows the entropy and the standard deviation based result comparison of the underexposed and the overexposed images. The processes implemented here are manually calculated and are implemented as per the required conditions which are based over the images received. The two types of images that were accepted for this algorithm is underexposed and the overexposed.

Table 4.1: Entropy after applying different techniques on overexposed images

\begin{tabular}{|c|c|}
\hline Entropy image tanlog & 4.3429 \\
\hline Entropy image Contrast Enhancement & 5.8552 \\
\hline Entropy image Median Filter & 5.8320 \\
\hline Entropy image Gamma Correction & 5.5536 \\
\hline Entropy image Manual Histogram Equalization & 5.4593 \\
\hline Entropy image after CDF & 5.5755 \\
\hline
\end{tabular}

Table 4.2: Entropy after applying different techniques on underexposed images

\begin{tabular}{|c|c|}
\hline Entropy image tanlog & 4.5187 \\
\hline Entropy image Contrast Enhancement & 4.8859 \\
\hline Entropy image Median Filter & 4.8959 \\
\hline Entropy image Gamma Correction & 4.6060 \\
\hline Entropy image Manual Histogram Equalization & 4.8506 \\
\hline Entropy image after homomorphic filter & 5.9105 \\
\hline Entropy after CDF & 6.1932 \\
\hline
\end{tabular}

Table 4.3: Absolute Standard deviation after applying different techniques on overexposed images

\begin{tabular}{l|l|}
\hline Absolute Standard deviation image tanlog & 50.9553 \\
\hline
\end{tabular}

Absolute Standard deviation image Contrast Enhancement 30.719

Absolute Standard deviation image Median Filter $\quad 31.2955$

Absolute Standard deviation image Gamma Correction 35.7457

Absolute Standard deviation image Manual Histogram Equalization

35.8905

\begin{tabular}{|c|c|}
\hline Absolute Standard deviation image tanlog & 13.5968 \\
\hline $\begin{array}{c}\text { Absolute Standard deviation image Contrast } \\
\text { Enhancement }\end{array}$ & 16.6961 \\
\hline Absolute Standard deviation image Median Filter & 16.9718 \\
\hline Absolute Standard deviation image Gamma Correction & 24.9976 \\
\hline $\begin{array}{c}\text { Absolute Standard deviation image Manual Histogram } \\
\text { Equalization }\end{array}$ & 31.604 \\
\hline Absolute Standard deviation image Homomorphic Filter & 22.808 \\
\hline Absolute Standard deviation after CDF & 30.8438 \\
\hline
\end{tabular}

Absolute Standard deviation image Homomorphic Filter 22.7573

Table 4.4: Absolute Standard deviation after applying different techniques on overexposed images

Below figures shows the subjective results indicating Overexposed input image and enhanced images. The output enhanced image contains more clear details and is more pleasing.

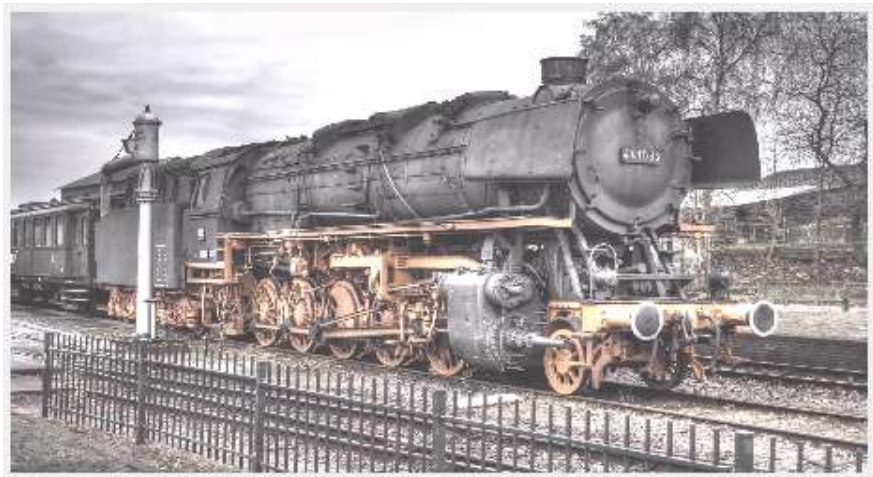

(a)

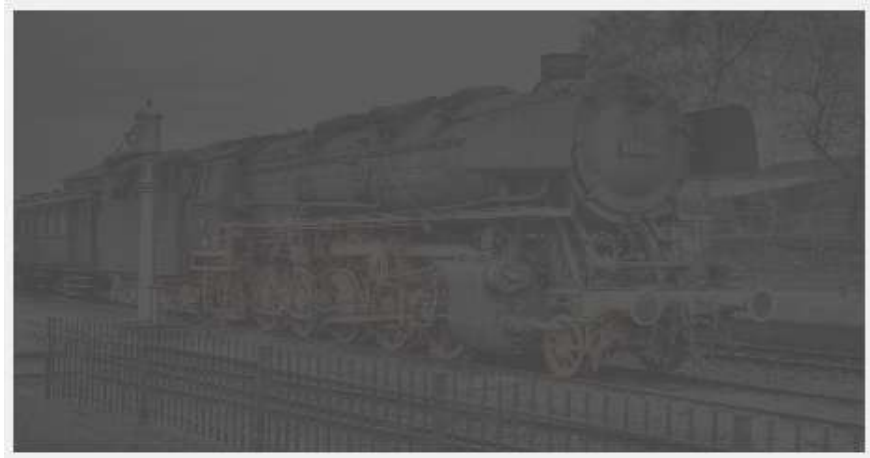

(b)

Volume 6 Issue 12, December 2017

\section{www.ijsr.net}

Licensed Under Creative Commons Attribution CC BY 


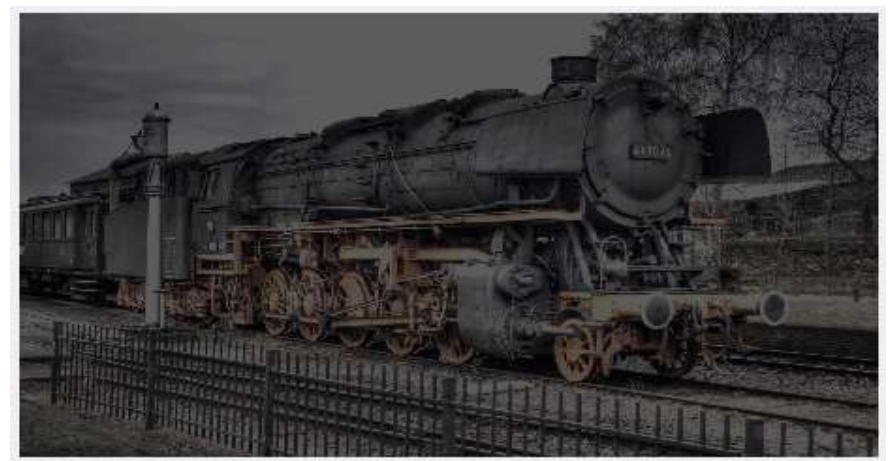

(c)

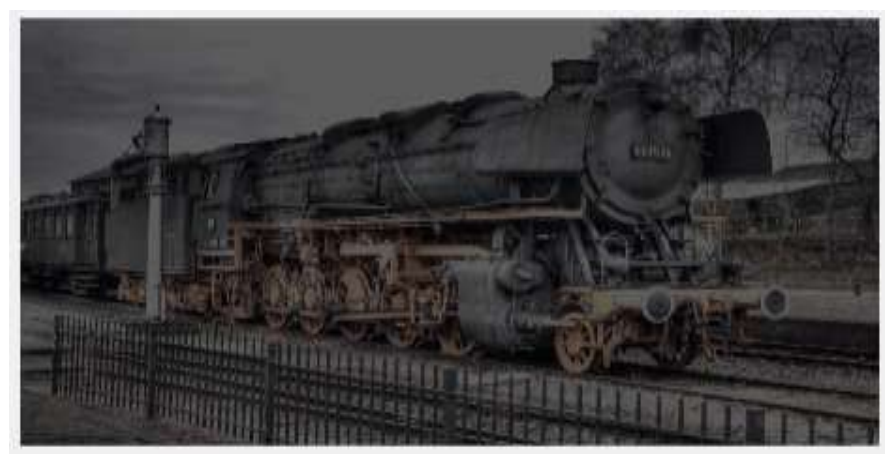

(d)

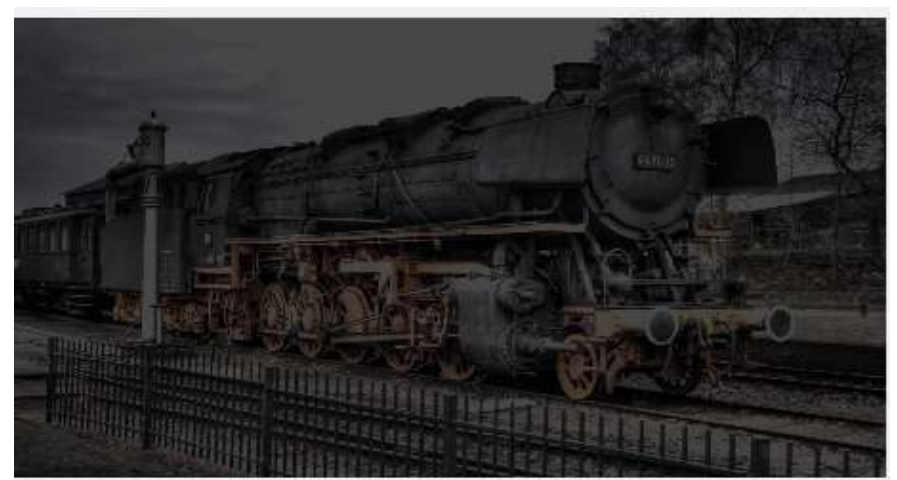

(e)

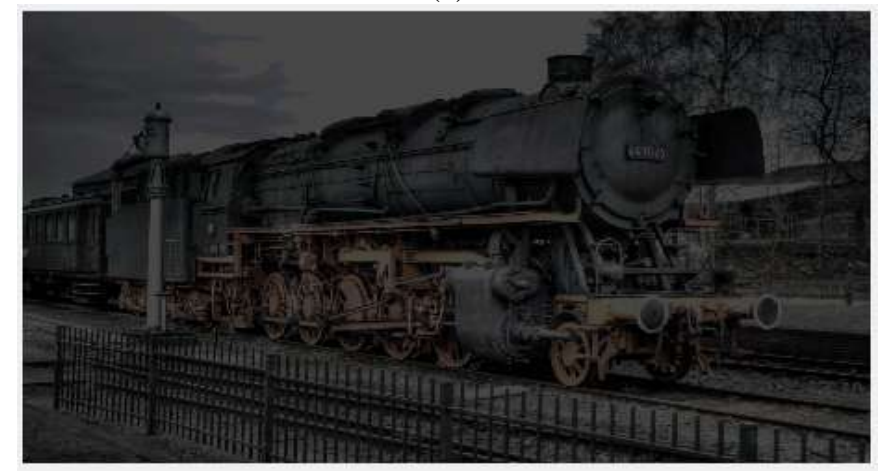

(f)

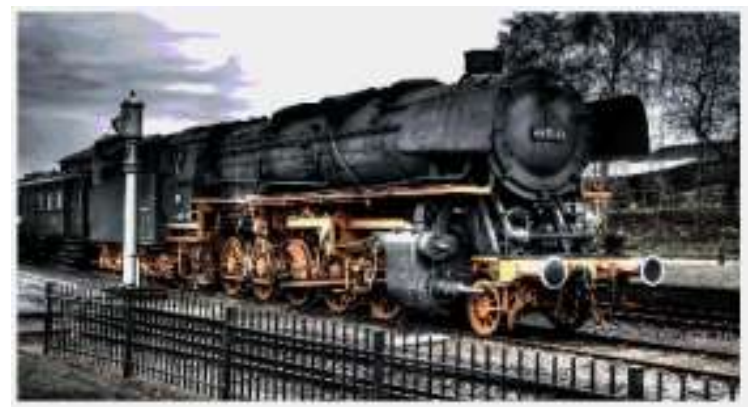

(g)

Figure 5.(a) Overexposed Input Image (b) Input Image after Tanlog process (c) Manual contrast enhanced image (d) Median filtered Image (e) Gamma Corrected Image (f)Manually Histogram Equalised Image (g) Output Image (Enhanced by Cumulative Distributive Function).

Now we will display and discuss the results on the basis of Histogram Equalization for Overexposed images. In below figure 6(a) the histogram equalization for input image is shown and it could be seen that histogram equalization of the input image is clustered towards 255 as input image is overexposed. But the processed output image shown in fig. 6(b) is distributed evenly so we can say that quality of image is improved.

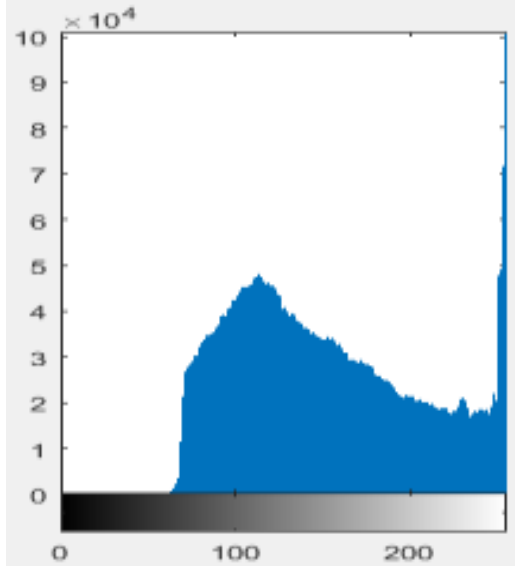

(a)

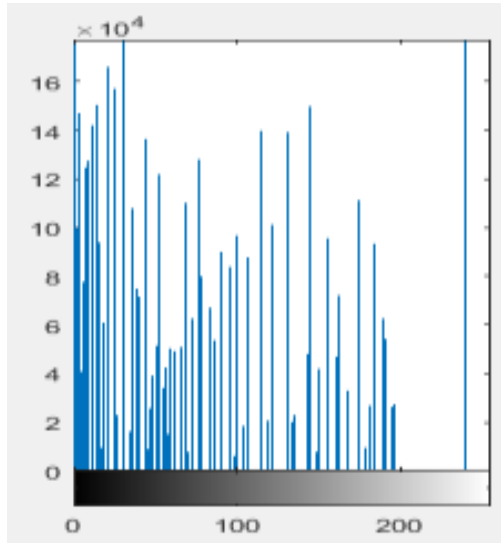

(b)

Figure 6: (a) Histogram of Input Image (Overexposed)

(b) Histogram of processed output Image

Volume 6 Issue 12, December 2017 www.ijsr.net 


\section{International Journal of Science and Research (IJSR) \\ ISSN (Online): 2319-7064}

Index Copernicus Value (2016): 79.57 | Impact Factor (2015): 6.391

For Overexposed Image the value of Peak Signal to Noise Ratio (PSNR) and Root Mean Square Error (RMSE) values are as shown below:-

PSNR of the input image with respect to original image = 11.3911

PSNRof the output image with respect to original image = 20.0744

RMSE of the input image with respect to original image = 68.39

RMSEof the output image with respect to original image $=$ 25.11

Structural Similarity Index (SSIM) value for image is as given below for overexposed input image :-

$$
\mathrm{SSIM}=0.7024
$$

Below figures shows the subjective results indicating underexposed input image and step by step enhanced images. The output enhanced image contains more clear details and is more pleasing.

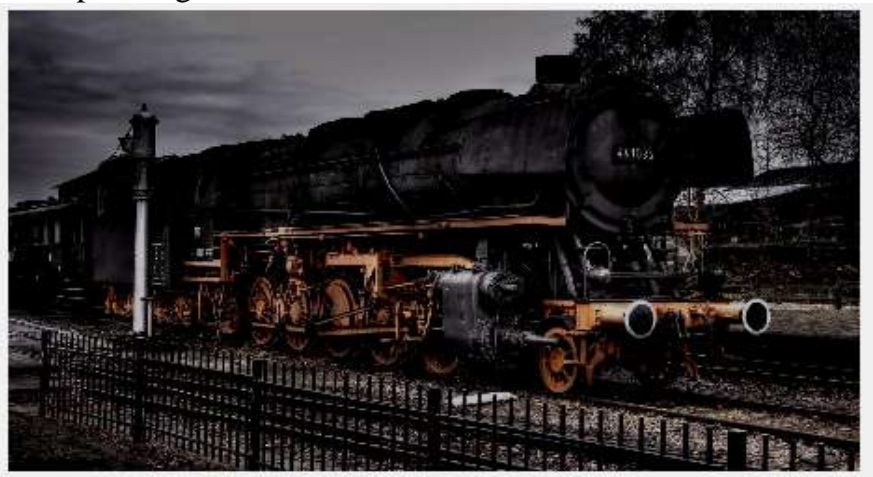

(a)

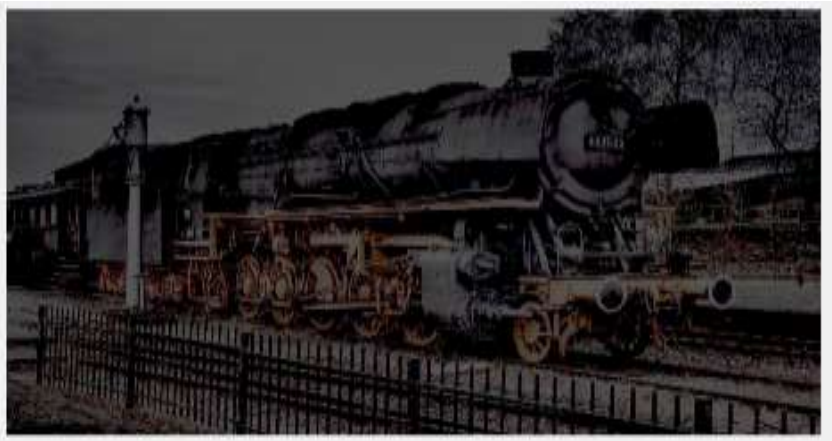

(b)

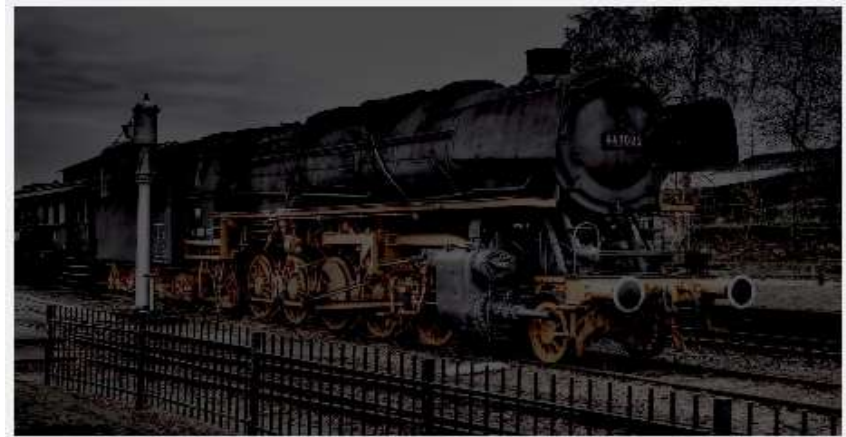

(c)

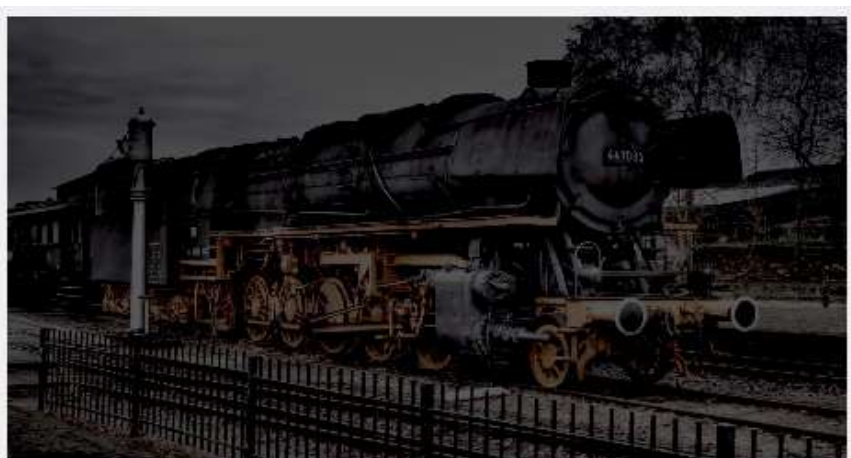

(d)

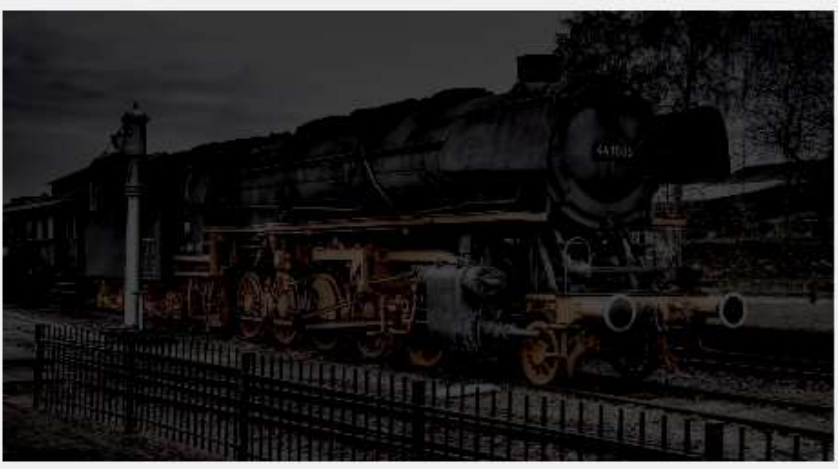

(e)

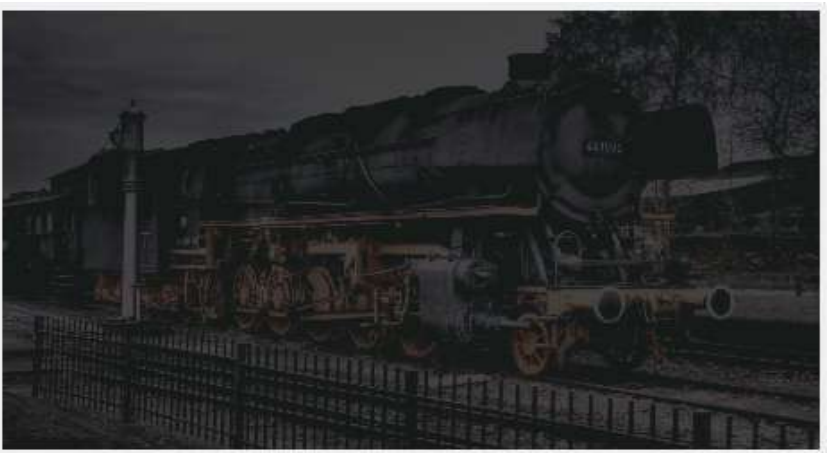

(f)

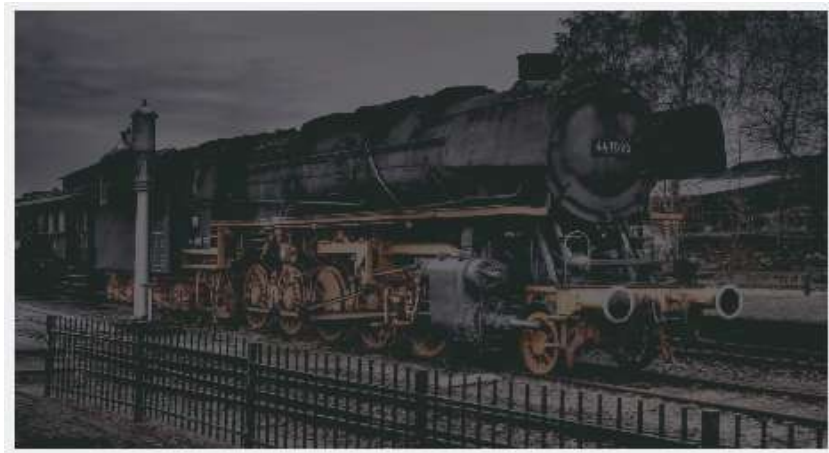

(g)

Volume 6 Issue 12, December 2017 www.ijsr.net 


\section{International Journal of Science and Research (IJSR) \\ ISSN (Online): 2319-7064}

Index Copernicus Value (2016): 79.57 | Impact Factor (2015): 6.391

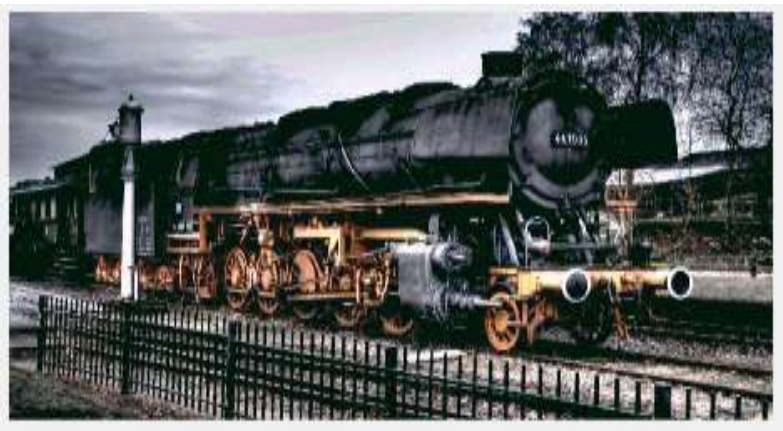

(h)

Figure 7: (a) Underexposed Input Image (b) Input Image after Tanlog process (c) Manual contrast enhanced image (d) Median filtered Image (e) Gamma Corrected Image (f)Manually Histogram Equalised Image (g) Homomorphic Filtered Image (h) Output Image (Enhanced by Cumulative Distributive Function)

Now we will display and discuss the results on the basis of Histogram Equalization for Underexposed images. In below figure 8(a) the histogram equalization for input image is shown and it could be seen that histogram equalization of the input image is clustered towards 0 as input image is underexposed. But the processed output image fig. 8(b) is distributed evenly so we can say that quality of image is improved.

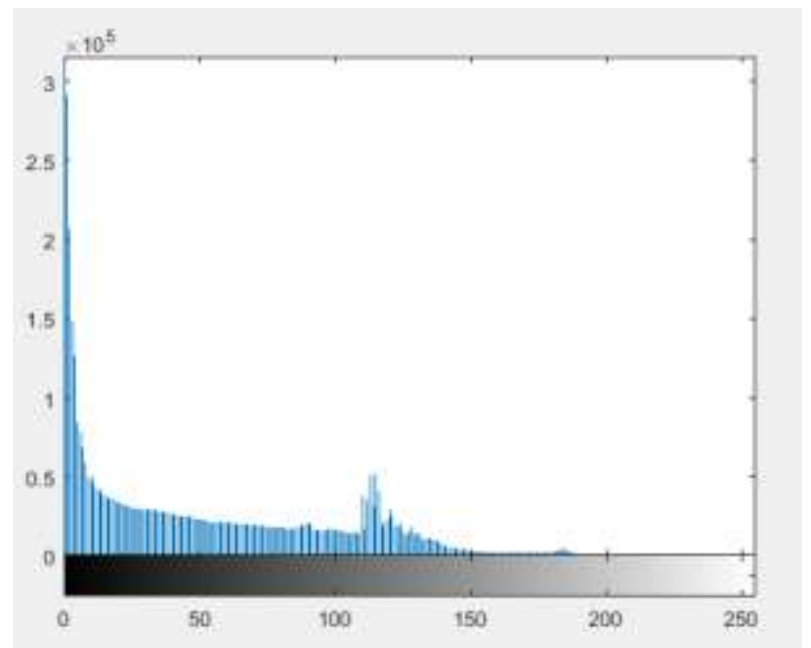

(a)

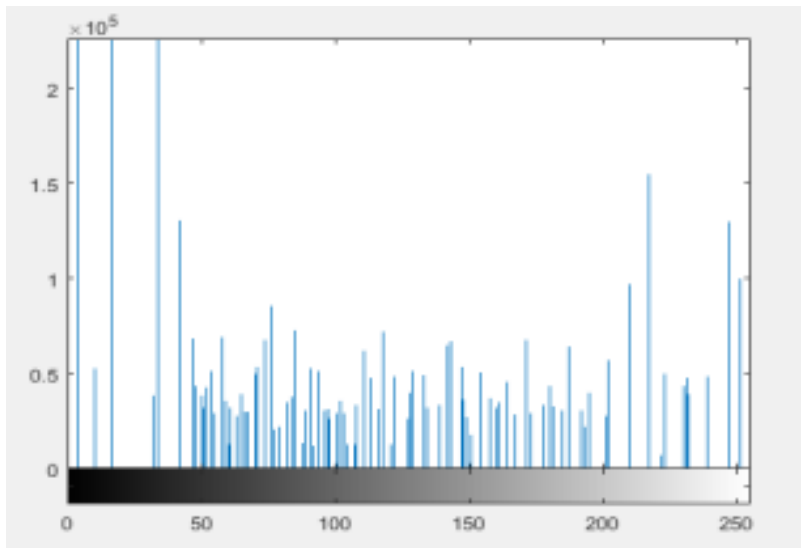

(b)

Figure 8: (a) Histogram of Input Image (Underexposed)

(b) Histogram of processed output Image

For Underexposed Image the value of Peak Signal to Noise Ratio (PSNR) and Mean Square Error (RMSE) values are as shown below:-

PSNR of the input image with respect to original image = 12.7715

PSNRof the output image with respect to original image = 19.4340

RMSE of the input image with respect to original image = 58.88

RMSEof the output image with respect to original image = 26.91

Structural Similarity Index (SSIM) value forunderexposed input image is as given below :-

$$
\text { SSIM }=0.5609
$$

\section{Conclusion}

The above presented results and the algorithm has shown some vital improvements for the field of the image enhancement algorithm, the other methodologies which were used earlier are dependent over the certain database of the images. The proposed work is improved in terms of the timing as well as it generates the output image in 0.03 seconds on an average basis which can further be improved by reducing the complexities of the filter algorithms such as the homomorphic filter and also the use of the different filters can also be considered as sometimes the image might exhibit the imaginary component missing as the image undergoes the Fast Fourier Transformation.

\section{References}

[1] Saravanan S. and P. Siva Kumar, "Image contrast enhancement using histogram equalization techniques: review," International Journal of Advances in Computer Science and Technology vol. 3, no. 3. March 2014.

[2] R. C. Gonzalez and R. E. Woods, Digital Image Processing. Reading, MA: Addison-Wesley, 1992.

[3] J. C. Russ, The Image Processing Handbook, CRC press, $5^{\text {th }}$ Ed., 2006. 


\section{International Journal of Science and Research (IJSR) \\ ISSN (Online): 2319-7064}

Index Copernicus Value (2016): 79.57 | Impact Factor (2015): 6.391

[4] N. Sengee, A. Sengee, and H.-K. Choi, "Image contrast enhancement using bi-histogram equalization with neighborhood metrics", IEEE Trans. on Consumer Electronics,

vol. 56, no. 4, pp. 2727-2734, November 2010.

[5] H.J.Zimmermann, "Fuzzy Set Theory and its Applications", Kluwer Academic Inc., 1991, ppl 1 -6

[6] K.S.Ravichandran ,P.Janani,J.Premaladha "image enhancement techniques: A Study" Indian Journal of Science and Technology", Vol 8(22) September 2015.

[7] Rajesh Garg, Bhawna Mittal,Sheetal Garg,"Histogram Equalization Techniques for Image Enhancement" IJECT Vol.2 Issue 1, March 2011,ISSN 2230-9543

[8] J.Premaladha "image enhancement techniques: A Study" Indian Journal of Science and Technology" ,Vol 8(22) September 2015.

[9] Bedi SS, Rati K. "Various image enhancement techniques: A critical review" International Journal of Advanced Research in Computer and Communication Engineering,Vol.2,2013.

[10] Dr.Muna F,Al-Samaraie "A new enahancement approach for enhancing image digital cameras by changing the contrast" International Journalof Advanced Science and Technology Vol.32,July 2011.

[11]Er. Mandeep Kaur,Er.Kiran Jain,Er.Virender Lather “ Study of image enhancement techniques:A Review" IJARCSSE, Vol.3 Issue 4,April 2013.

[12] Amanjot Kaur Randhawa ${ }^{1}$, Alka Bamotra ${ }^{2 " R e v i e w}$ Paper on Image Enhancement and Its Techniques"International Journal Of Engineering And Computer Science (IJECS), https://www.ijecs.in, Volume 6 Issue 5, May 2017, 21443-21447, DOI: $10.18535 / \mathrm{ijecs} / \mathrm{v} 6 \mathrm{i} 5.42$

Volume 6 Issue 12, December 2017 www.ijsr.net 